\title{
Assessment of Quality of Life in Radically Treated Head \& Neck Cancer Patients: A Cross Sectional Study
}

\author{
Shekhar Kumar Keshri, MBBS, DMRT, $M^{1^{*}}$, Satyendra Narayan Sinha, MBBS, DNB ${ }^{1}$ and Mitali Dan- \\ dekar Lall, MBBS, MS, DNB2 \\ ${ }^{1}$ Department of Radiation Oncology, Paras HMRI Hospital, India \\ ${ }^{2}$ Department of Surgical Oncology, Paras HMRI Hospital, India

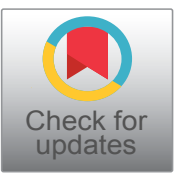

*Corresponding author: Shekhar Kumar Keshri, Senior Consultant, Department of Radiation Oncology, Paras HMRI Hospital, Patna, India, Tel: +91-9973043603

\begin{abstract}
Background: Head and neck cancers pose a significant healthcare burden in India and more than two third of these patients present in advance stage. Multimodality treatment is recommended and often has detrimental impact on quality of life.
\end{abstract}

Objectives: To assess the diverse domains of quality of life of radically treated head and neck cancer patients.

To discover the impact of treatment after completion (upto six weeks) and subsequent follow up irrespective of type of treatment delivered.

Materials and Methods: A cross-sectional study was conducted from February-May 2019 among radically treated 73 head and neck cancer patients attending OPD for follow up using EORTC QLQ-C30 and QLQ H\&N-35 questionnaires in Hindi language.

Results: Irrespective of the type of treatment received by patients as per their treatment plan on the basis of their diagnosis and staging (Either RT alone, Chemo-RT, Surgery followed by RT or, Surgery followed by Chemo-RT), analysis are done altogether. Taking both scales in account, nutritional supplements is only parameter found significant $(P=0.057)$ in all subgroups of patients. Factors which are found to have significant impact on QOL at different interval of follow up - Global Health Status, pain, nausea \& vomiting, appetite loss on QLQ-C30 and swallowing, senses \& speech problems, trouble with social contact \& eating, less sexuality, opening \& dry mouth, felt ill on QLQ H\&N-35. All had significant $p$-value.

Conclusion: Nutritional supplements are the significant factor in all subsets of treated head and neck cancer patients. Other above significant factors also need proper care and counselling to improve $\mathrm{QoL}$ in such patients.

\author{
Keywords \\ Quality of Life in Head and Neck Cancer, EORTC, QLQ \\ C-30, QLQ H\&N-35, QoL
}

\section{Introduction}

Head and neck cancers are common in India and account for about $30 \%$ of cancers in males and about $13 \%$ in females [1]. It poses a significant healthcare burden in India and more than two third of these patients present in advanced stage. Most of these patients require multidisciplinary management in combination of surgery, radiotherapy and chemotherapy. In spite of all treatment modalities used, the disease control rate for locally advanced head and neck cancer patients is about $40 \%$ at 5 years. Acute and Late toxicities of the treatment remain a challenge [2]. Myths, social taboos and lack of awareness in countries like India are still the main obstacle after diagnosis and during the treatment of cancers and thereafter [3]. Therefore, its management are associated with tremendous physiological, emotional, and psychological disruption. This affects patient's quality of life (QoL) significantly. What is Quality of Life? The world health organization defines quality of life as an "individual's perception of their position in life in the context of the culture and value system in which they live and in relation to their goals, expectations, standards and concerns" [4]. In other words, The Quality of life of an individual at a given time is the product of that person's inherent personality and the effect of transient factors such as disease and its treatment.

Citation: Keshri SK, Sinha SN, Lall MD (2020) Assessment of Quality of Life in Radically Treated Head \& Neck Cancer Patients: A Cross Sectional Study. Int J Oncol Res 3:022. doi.org/10.23937/26434563/1710022

Accepted: May 18, 2020; Published: May 20, 2020

Copyright: (c) 2020 Keshri SK, et al. This is an open-access article distributed under the terms of the Creative Commons Attribution License, which permits unrestricted use, distribution, and reproduction in any medium, provided the original author and source are credited. 
There are now a variety of well-validated health related - quality of life (HR-QoL) instruments available for measurements of QoL in the field of oncology. The term health related - quality of life (HR-QoL) is more disease specific and allows the health care professionals to focus upon the assessment of the impact of the disease and its treatment on physical, psychological and social aspect. The place of HR-QoL assessment in head and neck cancer practice has become more defined. It has had a major role in helping to shape the treatment strategies and patient support [4].

For this study, we chose the European Organization for Research and Treatment Quality of life Questionnaire based on EORTC QLQ-C30 and EORTC QLQ H\&N35 to measure QoL in our head and neck cancer patients because it has been widely validated in clinical trials and has been translated and linguistically validated into more than 60 languages including Hindi $[5,6]$.

\section{Objectives}

- To assess the diverse domains of quality of life of radically treated head and neck cancer patients.

- To discover the impact of treatment after completion and subsequent follow up irrespective of type of treatment delivered.

\section{Materials and Methods}

This is a cross-sectional study conducted at Paras Cancer Centre, Paras HMRI, Patna (India) over three months between February and May 2019 among radically treated head and neck cancer patients attending radiation oncology OPD for follow up after completion of their treatment.

\section{Inclusion criteria}

- Patients who suffered of head and neck cancer only.

- Patients who had been radically treated for head and neck cancers and all were attending the oncology OPD for follow-up during this period.

\section{Exclusion criteria}

- Patients who had cancers other than head and neck cancers,

- Patients requiring palliative treatment for the proven head and neck cancers,

- Patient with recurrent disease,

- Patients who were reluctant to provide information and,

- Patients diagnosed with any second primary were excluded.

We have used the Hindi version of standard EORTC Questionnaires QLQ C-30 (version 3.0) and QLQ H\&N-35 (version 1.0) as provided by the EORTC (to conduct the study permission taken from EORTC). Patients received the questionnaires sheet while waiting for routine check-up in the outpatient department, were explained in detail about the sheet and asked to fill.

The QLQ-C30 is composed of both multi-item scales and single-item measures. These include five functional scales, three symptom scales, a global health status/ QoL scale, and six single items. Each of the multi-item scales includes a different set of items - no item occurs in more than one scale.

All of the scales and single-item measures range in score from 0 to 100. A high scale score represents a higher response level.

Thus, a high score for a functional scale represents a high/healthy level of functioning, a high score for the global health status/QoL represents a high QoL, but a high score for a symptom scale/item represents a high level of symptomatology/problems.

The head \& neck cancer module incorporates seven multi-item scales that assess pain, swallowing, senses (taste and smell), speech, social eating, social contact and sexuality. There are also eleven single items. For all items and scales, high scores indicate more problems (i.e. there are no function scales in which high scores would mean better functioning). The scoring approach for the QLQ-H\&N35 is identical in principle to that for the symptom scales/single items of the QLQ-C30.

The scoring was done as per the EORTC scoring manual as described below:

For all scales, the Raw Score, RS, is the mean of the component items:

Raw Score $=$ RS $=\left(I_{1}+I_{2}+\ldots .+I_{n}\right) / \mathrm{n}$

Then for Functional Scales:

Score $=1-\left\{\frac{(R S-1)}{\text { range }}\right\} \times 100$

And for Symptom scales/items and Global health status/QoL:

Score $=\{(\mathrm{RS}-1) / \text { range }\}^{*} 100$

\section{Statistical analysis}

Data was entered in Microsoft excel and student $t$ Test and One-way ANOVA test was analyzed by using statistical analysis software Graphpad Prism (Version 5). The data were presented as Mean \pm SD. ' $p$ ' value of less than 0.05 was accepted as indicating significance.

\section{Results}

A total of 73 head and neck cancer patients as per inclusion criteria (Male - 60 \& Female - 13) took part in the study during above period of time of over three months. 72 patients were married, and 1 patient was unmarried. $63 \%$ patients were in age group $41-60$ years. Oral cavity constitutes the most common location (83.6\%). $76.7 \%$ patients were in state III \& IV (Table 1 and Table 2). 
From Tables 3, we see that on the EORTC QLQ C-30 scale, the overall QoL showed non-significant difference between the two groups: Early staged tumors (stages I and II) and advanced-staged tumors (stages III and IV) except dyspnea ( $p=0.0053)$ and constipation ( $p=$ 0.0264 ) which had better scores in early staged tumors. Symptom like swallowing and dry mouth had better score in early stage tumors on the QLQ H\&N-35 (Table 3).

Table 1: Distribution of the respondents according to the sociodemographic characteristics $(\mathrm{N}=73)$.

\begin{tabular}{|l|l|}
\hline Characteristic & Frequency n (\%) \\
\hline Age & \multicolumn{1}{l|}{$14(19.18 \%)$} \\
\hline $41-40$ & $46(63.01 \%)$ \\
\hline$>60$ & $13(17.81 \%)$ \\
\hline Gender & \\
\hline Male & $60(82.19 \%)$ \\
\hline Female & $13(17.81 \%)$ \\
\hline Occupation & \\
\hline Farmer & $18(24.66 \%)$ \\
\hline Housewife & $11(15.07 \%)$ \\
\hline Business & $19(26.03 \%)$ \\
\hline Daily wage worker & $5(6.85 \%)$ \\
\hline Salary based worker/Professional & $20(27.40 \%)$ \\
\hline Education & \\
\hline Illiterate & $10(13.70 \%)$ \\
\hline Up to 10 & $30(41.10 \%)$ \\
\hline Secondary & $15(20.55 \%)$ \\
\hline Graduate & $15(20.55 \%)$ \\
\hline Postgraduate & $3(4.11 \%)$ \\
\hline Marital Status & $1(1.37 \%)$ \\
\hline Single & $72(98.63 \%)$ \\
\hline Married & \\
\hline & \\
\hline & \\
\hline
\end{tabular}

Modalities of the treatment received by the patients are either RT alone $(n=3)$, Chemo RT alone $(n=8)$, Surgery followed by Chemo RT ( $n=12)$ or, Surgery followed by RT $(n=50)$ as per plan on the basis of their diagnosis and staging. Nutritional supplement is the only parameter which was found to be significant as a parameter in

Table 2: Distribution of cancer patients according to cancer site, stage, type of treatment and time point of interview $(\mathrm{N}=$ 73).

\begin{tabular}{|l|l|}
\hline Characteristics & \multicolumn{2}{l|}{ Frequency n (\%) } \\
\hline Cancer site & $2(2.74 \%)$ \\
\hline Larynx & $61(83.56 \%)$ \\
\hline Oral Cavity & $7(9.59 \%)$ \\
\hline Oropharynx & $3(4.11 \%)$ \\
\hline Paranasal Sinus & \\
\hline Cancer stage & $1(1.37 \%)$ \\
\hline Stage 1 & $16(21.92 \%)$ \\
\hline Stage 2 & $25(34.25 \%)$ \\
\hline Stage 3 & $31(42.47 \%)$ \\
\hline Stage 4 & \\
\hline Treatment type & $3(4.11 \%)$ \\
\hline RT & $8(10.96 \%)$ \\
\hline CT + RT & $50(68.49 \%)$ \\
\hline Surgery + RT & $12(16.44 \%)$ \\
\hline Surgery + CT + RT & $8(10.96 \%)$ \\
\hline Time gap between completion of treatment and \\
researcher's interview & $3(4.11 \%)$ \\
\hline$<1$ month & $6(8.22 \%)$ \\
\hline 1-3 month & $32(43.84 \%)$ \\
\hline 3-6 months & $11(15.07 \%)$ \\
\hline 6-12 months & $13(17.81 \%)$ \\
\hline 12-24 months & \\
\hline > 24 months & \\
\hline & \\
\hline
\end{tabular}

Table 3: Early stage tumors vs. late-stage tumors $(N=73)$ QLQ C-30 \& QLQH \& N-35.

\begin{tabular}{|l|l|l|l|}
\hline \multirow{2}{*}{ Scales (QLQ-C30) } & \multicolumn{2}{l|}{ Stage } & student t Test \\
\cline { 2 - 3 } & $\mathrm{I} / \mathrm{II}(\mathrm{n}=17)$ & $\mathrm{II} / \mathrm{IV}(\mathrm{n}=56)$ & P value \\
\hline Global health status & $61.76 \pm 28.27$ & $68.75 \pm 22.2$ & 0.2909 \\
\hline Physical functioning & $89.8 \pm 10.83$ & $85.6 \pm 16.52$ & 0.3276 \\
\hline Role functioning & $90.2 \pm 16.73$ & $87.5 \pm 22.53$ & 0.6499 \\
\hline Emotional functioning & $81.37 \pm 21.96$ & $73.66 \pm 24.91$ & 0.2552 \\
\hline Cognitive functioning & $85.29 \pm 26.27$ & $89.58 \pm 18.14$ & 0.447 \\
\hline Social functioning & $78.43 \pm 30.48$ & $88.99 \pm 20.91$ & 0.1079 \\
\hline Fatigue & $22.88 \pm 15.45$ & $29.96 \pm 22.32$ & 0.2264 \\
\hline Nausea and vomiting & $2.941 \pm 6.549$ & $3.571 \pm 9.382$ & 0.7972 \\
\hline Pain & $17.65 \pm 27.93$ & $17.56 \pm 21.18$ & 0.989 \\
\hline Dyspnoea & $9.804 \pm 19.6$ & $1.19 \pm 6.242$ & 0.0053 \\
\hline Insomnia & $17.65 \pm 29.15$ & $16.07 \pm 22.91$ & 0.8167 \\
\hline Appetite loss & $25.49 \pm 34.42$ & $20.24 \pm 29.6$ & 0.5393 \\
\hline
\end{tabular}




\begin{tabular}{|c|c|c|c|}
\hline Constipation & $25.49 \pm 25.08$ & $11.9 \pm 20.53$ & 0.0264 \\
\hline Diarrhoea & $3.922 \pm 11.07$ & $4.167 \pm 12.81$ & 0.9435 \\
\hline Financial difficulties & $58.82 \pm 38.24$ & $52.38 \pm 35.31$ & 0.5201 \\
\hline \multicolumn{4}{|l|}{ QLQ H\&N-35 } \\
\hline Pain & $21.08 \pm 28.73$ & $16.82 \pm 17.59$ & 0.4580 \\
\hline Swallowing & $19.61 \pm 21.84$ & $26.04 \pm 20.72$ & 0.2718 \\
\hline Senses problems & $17.65 \pm 21.63$ & $19.35 \pm 18.74$ & 0.7532 \\
\hline Speech problems & $26.8 \pm 29.15$ & $26.59 \pm 21.37$ & 0.9742 \\
\hline Trouble with social eating & $34.8 \pm 33.36$ & $29.32 \pm 26.92$ & 0.4890 \\
\hline Trouble with social contact & $26.27 \pm 31.93$ & $18.33 \pm 21.32$ & 0.2384 \\
\hline Less sexuality & $15.69 \pm 29.15$ & $20.24 \pm 30.77$ & 0.5905 \\
\hline Teeth & $33.33 \pm 33.33$ & $32.14 \pm 29.79$ & 0.8888 \\
\hline Opening mouth & $45.1 \pm 35.24$ & $41.07 \pm 37.6$ & 0.6961 \\
\hline Dry mouth & $37.25 \pm 35.12$ & $50.6 \pm 33.63$ & 0.1605 \\
\hline Sticky saliva & $29.41 \pm 35.12$ & $30.95 \pm 32.32$ & 0.8665 \\
\hline Coughing & $17.65 \pm 20.81$ & $14.88 \pm 18.98$ & 0.6084 \\
\hline Felt ill & $23.53 \pm 28.3$ & $30.95 \pm 29.72$ & 0.3650 \\
\hline Pain killers & $23.53 \pm 43.72$ & $21.43 \pm 41.4$ & 0.8570 \\
\hline Nutritional supplements & $35.29 \pm 49.26$ & $21.43 \pm 41.4$ & 0.2514 \\
\hline Feeding tube & $0 \pm 0$ & $1.786 \pm 13.36$ & NA \\
\hline Weight loss & $41.18 \pm 50.73$ & $17.86 \pm 38.65$ & 0.0471 \\
\hline Weight gain & $29.41 \pm 46.97$ & $55.36 \pm 50.16$ & 0.0623 \\
\hline
\end{tabular}

Table 4: Impact of type of treatment on QOL scores with EORTC QLQ-30 \& QLQ H\&N-35 questionnaires.

\begin{tabular}{|c|c|c|c|c|c|}
\hline Scales (QLQ-C30) & $C T+R T(n=8)$ & $\mathrm{RT}(\mathrm{n}=3)$ & $S X+C T+R T(n=12)$ & $S X+R T(n=50)$ & ANOVA $P$ value \\
\hline Global health status & $54.17 \pm 22.71$ & $72.22 \pm 25.46$ & $64.58 \pm 11.31$ & $69.50 \pm 25.73$ & 0.3732 \\
\hline Physical functioning & $87.50 \pm 12.05$ & $84.44 \pm 26.94$ & $94.44 \pm 6.25$ & $84.67 \pm 16.45$ & 0.2668 \\
\hline Role functioning & $85.42 \pm 16.52$ & $83.33 \pm 28.87$ & $95.83 \pm 10.36$ & $87.00 \pm 23.40$ & 0.5806 \\
\hline Emotional functioning & $71.88 \pm 18.87$ & $75.00 \pm 36.32$ & $88.19 \pm 12.54$ & $73.00 \pm 26.11$ & 0.2675 \\
\hline Cognitive functioning & $89.58 \pm 15.27$ & $83.33 \pm 28.87$ & $95.83 \pm 10.36$ & $87.00 \pm 22.15$ & 0.5656 \\
\hline Social functioning & $85.42 \pm 20.77$ & $66.67 \pm 44.10$ & $91.67 \pm 16.67$ & $86.67 \pm 24.28$ & 0.4485 \\
\hline Fatigue & $33.33 \pm 18.78$ & $29.63 \pm 27.96$ & $25.93 \pm 15.95$ & $28.00 \pm 22.47$ & 0.8933 \\
\hline Nausea and vomiting & $4.17 \pm 7.72$ & $5.56 \pm 9.62$ & $4.17 \pm 10.36$ & $3.00 \pm 8.71$ & 0.9352 \\
\hline Pain & $22.92 \pm 23.46$ & $27.78 \pm 34.69$ & $11.11 \pm 14.79$ & $17.67 \pm 23.67$ & 0.5772 \\
\hline Dyspnoea & $12.50 \pm 24.80$ & $0.00 \pm 0.00$ & $0.00 \pm 0.00$ & $2.67 \pm 9.14$ & 0.0794 \\
\hline Insomnia & $25.00 \pm 34.50$ & $33.33 \pm 33.33$ & $11.11 \pm 21.71$ & $15.33 \pm 22.55$ & 0.3789 \\
\hline Appetite loss & $25.00 \pm 23.57$ & $33.33 \pm 33.33$ & $27.78 \pm 39.78$ & $18.67 \pm 29.48$ & 0.6942 \\
\hline Constipation & $25.00 \pm 15.43$ & $11.11 \pm 19.24$ & $11.11 \pm 21.71$ & $14.67 \pm 23.48$ & 0.5638 \\
\hline Diarrhoea & $0.00 \pm 0.00$ & $0.00 \pm 0.00$ & $2.78 \pm 9.62$ & $5.33 \pm 14.06$ & 0.6136 \\
\hline Financial difficulties & $41.67 \pm 29.55$ & $44.44 \pm 50.92$ & $55.56 \pm 32.82$ & $56.00 \pm 37.16$ & 0.7283 \\
\hline \multicolumn{6}{|l|}{ QLQ H\&N-35 } \\
\hline Pain & $15.63 \pm 13.68$ & $41.67 \pm 46.40$ & $13.19 \pm 9.70$ & $17.83 \pm 21.10$ & 0.1933 \\
\hline Swallowing & $17.71 \pm 12.15$ & $30.56 \pm 33.68$ & $31.94 \pm 16.98$ & $23.50 \pm 22.19$ & 0.4470 \\
\hline Senses problems & $14.58 \pm 13.91$ & $16.67 \pm 16.67$ & $26.39 \pm 18.06$ & $18.00 \pm 20.44$ & 0.5107 \\
\hline Speech problems & $27.78 \pm 23.00$ & $33.33 \pm 33.33$ & $20.37 \pm 21.10$ & $27.56 \pm 23.57$ & 0.7548 \\
\hline Trouble with social eating & $29.17 \pm 30.21$ & $27.78 \pm 25.46$ & $28.47 \pm 20.55$ & $31.50 \pm 30.50$ & 0.9835 \\
\hline Trouble with social contact & $15.00 \pm 25.13$ & $22.22 \pm 38.49$ & $17.78 \pm 12.50$ & $21.47 \pm 25.81$ & 0.8899 \\
\hline Less sexuality & $25.00 \pm 38.83$ & $11.11 \pm 19.24$ & $16.67 \pm 18.80$ & $19.33 \pm 32.01$ & 0.9021 \\
\hline Teeth & $29.17 \pm 27.82$ & $22.22 \pm 38.49$ & $16.67 \pm 17.41$ & $37.33 \pm 32.04$ & 0.1753 \\
\hline Opening mouth & $33.33 \pm 30.86$ & $33.33 \pm 33.33$ & $47.22 \pm 26.43$ & $42.67 \pm 40.43$ & 0.8398 \\
\hline
\end{tabular}




\begin{tabular}{|l|l|l|l|l|l|}
\hline Dry mouth & $41.67 \pm 34.50$ & $55.56 \pm 38.49$ & $52.78 \pm 33.21$ & $46.67 \pm 34.99$ & 0.8749 \\
\hline Sticky saliva & $25.00 \pm 23.57$ & $44.44 \pm 50.92$ & $36.11 \pm 36.12$ & $29.33 \pm 32.74$ & 0.7643 \\
\hline Coughing & $16.67 \pm 17.82$ & $22.22 \pm 38.49$ & $11.11 \pm 16.41$ & $16.00 \pm 19.33$ & 0.7971 \\
\hline Felt ill & $29.17 \pm 27.82$ & $33.33 \pm 33.33$ & $19.44 \pm 22.29$ & $31.33 \pm 31.16$ & 0.6575 \\
\hline Pain killers & $50.00 \pm 53.45$ & $33.33 \pm 57.74$ & $33.33 \pm 49.24$ & $14.00 \pm 35.05$ & 0.0840 \\
\hline Nutritional supplements & $62.50 \pm 51.75$ & $33.33 \pm 57.74$ & $25.00 \pm 45.23$ & $18.00 \pm 38.81$ & 0.0572 \\
\hline Feeding tube & $0.00 \pm 0.00$ & $0.00 \pm 0.00$ & $8.33 \pm 28.87$ & $0.00 \pm 0.00$ & 0.1655 \\
\hline Weight loss & $37.50 \pm 51.75$ & $33.33 \pm 57.74$ & $16.67 \pm 38.92$ & $22.00 \pm 41.85$ & 0.7149 \\
\hline Weight gain & $37.50 \pm 51.75$ & $0.00 \pm 0.00$ & $58.33 \pm 51.49$ & $52.00 \pm 50.47$ & 0.2820 \\
\hline
\end{tabular}

Table 5: Mean Scores distributed across different categories of interview time with EORTC QLQ C-30 (N = 73).

\begin{tabular}{|c|c|c|c|c|c|c|c|}
\hline \multirow{2}{*}{ Scales (QLQ-C30) } & \multirow{2}{*}{$\begin{array}{l}<1 \text { month } \\
(n=6)\end{array}$} & \multirow{2}{*}{$\begin{array}{l}1-3 \text { months } \\
(n=32)\end{array}$} & \multirow{2}{*}{$\begin{array}{l}\text { 3-6 months } \\
(n=11)\end{array}$} & \multirow{2}{*}{$\begin{array}{l}\text { 6-12 months } \\
(n=13)\end{array}$} & \multirow{2}{*}{$\begin{array}{l}12-24 \text { months } \\
(\mathrm{n}=8)\end{array}$} & \multirow{2}{*}{$\begin{array}{l}>24 \text { months } \\
(n=3)\end{array}$} & \multirow{2}{*}{$\begin{array}{l}\text { ANOVA } \\
\text { P value }\end{array}$} \\
\hline & & & & & & & \\
\hline $\begin{array}{l}\text { Global health } \\
\text { status }\end{array}$ & $52.78 \pm 38.25$ & $62.76 \pm 18.33$ & $62.88 \pm 27.22$ & $71.79 \pm 20.84$ & $89.58 \pm 17.68$ & $77.78 \pm 25.46$ & 0.03 \\
\hline $\begin{array}{l}\text { Physical } \\
\text { functioning }\end{array}$ & $77.78 \pm 25.88$ & $84.58 \pm 17.22$ & $83.03 \pm 12.78$ & $91.79 \pm 7.77$ & $94.17 \pm 6.61$ & $95.56 \pm 7.70$ & 0.1827 \\
\hline Role functioning & $83.33 \pm 33.33$ & $84.38 \pm 25.38$ & $86.36 \pm 16.36$ & $96.15 \pm 9.99$ & $95.83 \pm 7.72$ & $88.89 \pm 19.24$ & 0.5197 \\
\hline $\begin{array}{l}\text { Emotional } \\
\text { functioning }\end{array}$ & $66.67 \pm 34.96$ & $73.18 \pm 23.83$ & $69.70 \pm 25.08$ & $78.21 \pm 24.42$ & $90.63 \pm 12.15$ & $86.11 \pm 24.06$ & 0.3609 \\
\hline $\begin{array}{l}\text { Cognitive } \\
\text { functioning }\end{array}$ & $86.11 \pm 34.02$ & $84.90 \pm 24.08$ & $89.39 \pm 15.41$ & $93.59 \pm 10.84$ & $91.67 \pm 8.91$ & $100.00 \pm 0.00$ & 0.6952 \\
\hline Social functioning & $100.00 \pm 0.00$ & $83.85 \pm 29.17$ & $83.33 \pm 23.57$ & $88.46 \pm 17.19$ & $85.42 \pm 20.77$ & $94.44 \pm 9.62$ & 0.7146 \\
\hline Fatigue & $35.19 \pm 17.80$ & $31.25 \pm 19.23$ & $37.37 \pm 26.42$ & $20.51 \pm 19.16$ & $16.67 \pm 17.82$ & $14.81 \pm 25.66$ & 0.113 \\
\hline $\begin{array}{l}\text { Nausea and } \\
\text { vomiting }\end{array}$ & $19.44 \pm 16.39$ & $2.60 \pm 7.47$ & $1.52 \pm 5.03$ & $0.00 \pm 0.00$ & $4.17 \pm 7.72$ & $0.00 \pm 0.00$ & $<0.0001$ \\
\hline Pain & $38.89 \pm 43.03$ & $21.35 \pm 22.49$ & $18.18 \pm 18.94$ & $10.26 \pm 10.84$ & $4.17 \pm 11.79$ & $0.00 \pm 0.00$ & 0.0274 \\
\hline Dyspnoea & $0.00 \pm 0.00$ & $4.17 \pm 14.04$ & $6.06 \pm 13.48$ & $0.00 \pm 0.00$ & $4.17 \pm 11.79$ & $0.00 \pm 0.00$ & 0.7521 \\
\hline Insomnia & $38.89 \pm 38.97$ & $18.75 \pm 25.31$ & $18.18 \pm 22.92$ & $7.69 \pm 14.62$ & $8.33 \pm 15.43$ & $0.00 \pm 0.00$ & 0.0874 \\
\hline Appetite loss & $50.00 \pm 45.95$ & $18.75 \pm 28.00$ & $36.36 \pm 37.87$ & $15.38 \pm 22.01$ & $8.33 \pm 15.43$ & $0.00 \pm 0.00$ & 0.0355 \\
\hline Constipation & $11.11 \pm 17.21$ & $15.62 \pm 22.38$ & $15.15 \pm 31.14$ & $10.26 \pm 21.01$ & $16.67 \pm 17.82$ & $33.33 \pm 0.00$ & 0.7314 \\
\hline Diarrhoea & $5.56 \pm 13.61$ & $2.08 \pm 11.79$ & $12.12 \pm 16.82$ & $2.56 \pm 9.25$ & $4.17 \pm 11.79$ & $0.00 \pm 0.00$ & 0.2998 \\
\hline $\begin{array}{l}\text { Financial } \\
\text { difficulties }\end{array}$ & $33.33 \pm 29.81$ & $57.29 \pm 35.15$ & $57.58 \pm 39.70$ & $58.97 \pm 33.76$ & $45.83 \pm 39.59$ & $44.44 \pm 50.92$ & 0.6746 \\
\hline \multicolumn{8}{|l|}{ QLQ H\&N-35 } \\
\hline Pain & $47.22 \pm 37.14$ & $19.79 \pm 18.42$ & $19.70 \pm 16.36$ & $8.33 \pm 9.62$ & $7.29 \pm 11.30$ & $0.00 \pm 0.00$ & 0.0005 \\
\hline Swallowing & $52.78 \pm 27.72$ & $26.04 \pm 18.18$ & $26.52 \pm 18.57$ & $19.87 \pm 19.99$ & $10.42 \pm 13.18$ & $2.78 \pm 4.81$ & 0.0010 \\
\hline Senses problems & $44.44 \pm 20.18$ & $22.92 \pm 18.81$ & $15.15 \pm 17.41$ & $14.10 \pm 14.98$ & $2.08 \pm 5.89$ & $5.56 \pm 9.62$ & 0.0003 \\
\hline Speech problems & $53.70 \pm 34.01$ & $27.08 \pm 20.73$ & $33.33 \pm 18.59$ & $21.37 \pm 21.97$ & $12.50 \pm 16.20$ & $3.70 \pm 6.42$ & 0.0048 \\
\hline $\begin{array}{l}\text { Trouble with social } \\
\text { eating }\end{array}$ & $68.06 \pm 33.51$ & $30.47 \pm 24.56$ & $40.91 \pm 35.83$ & $19.87 \pm 17.85$ & $16.67 \pm 17.25$ & $2.78 \pm 4.81$ & 0.0011 \\
\hline $\begin{array}{l}\text { Trouble with social } \\
\text { contact }\end{array}$ & $45.56 \pm 41.83$ & $19.58 \pm 20.73$ & $30.91 \pm 29.10$ & $12.82 \pm 15.98$ & $7.50 \pm 7.51$ & $2.22 \pm 3.85$ & 0.0121 \\
\hline Less sexuality & $58.33 \pm 46.84$ & $16.15 \pm 24.86$ & $28.79 \pm 40.89$ & $14.10 \pm 19.06$ & $4.17 \pm 11.79$ & $0.00 \pm 0.00$ & 0.0068 \\
\hline Teeth & $27.78 \pm 44.31$ & $36.46 \pm 33.18$ & $39.39 \pm 35.96$ & $28.20 \pm 12.52$ & $25.00 \pm 23.57$ & $11.11 \pm 19.24$ & 0.6396 \\
\hline Opening mouth & $77.78 \pm 27.22$ & $40.62 \pm 35.66$ & $42.42 \pm 42.40$ & $48.72 \pm 32.25$ & $16.67 \pm 35.63$ & $22.22 \pm 19.24$ & 0.0495 \\
\hline Dry mouth & $61.11 \pm 38.97$ & $51.04 \pm 32.77$ & $42.42 \pm 36.79$ & $61.54 \pm 32.90$ & $20.83 \pm 17.25$ & $11.11 \pm 19.24$ & 0.0290 \\
\hline Sticky saliva & $61.11 \pm 38.97$ & $38.54 \pm 31.80$ & $18.18 \pm 27.34$ & $25.64 \pm 33.76$ & $12.50 \pm 17.25$ & $0.00 \pm 0.00$ & 0.0111 \\
\hline Coughing & $22.22 \pm 17.21$ & $12.50 \pm 18.45$ & $21.21 \pm 22.47$ & $12.82 \pm 21.68$ & $16.67 \pm 17.82$ & $22.22 \pm 19.24$ & 0.6971 \\
\hline Felt ill & $61.11 \pm 32.77$ & $30.21 \pm 27.25$ & $39.39 \pm 35.96$ & $17.95 \pm 22.01$ & $12.50 \pm 17.25$ & $11.11 \pm 19.24$ & 0.0108 \\
\hline Pain killers & $16.67 \pm 40.82$ & $31.25 \pm 47.09$ & $9.09 \pm 30.15$ & $23.08 \pm 43.85$ & $12.50 \pm 35.36$ & $0.00 \pm 0.00$ & 0.5627 \\
\hline
\end{tabular}




\begin{tabular}{|l|l|l|l|l|l|l|l|}
\hline $\begin{array}{l}\text { Nutritional } \\
\text { supplements }\end{array}$ & $50.00 \pm 54.77$ & $25.00 \pm 43.99$ & $27.27 \pm 46.71$ & $15.38 \pm 37.55$ & $0.00 \pm 0.00$ & $66.67 \pm 57.74$ & 0.1485 \\
\hline Feeding tube & $0.00 \pm 0.00$ & $0.00 \pm 0.00$ & $9.09 \pm 30.15$ & $0.00 \pm 0.00$ & $0.00 \pm 0.00$ & $0.00 \pm 0.00$ & 0.3490 \\
\hline Weight loss & $83.33 \pm 40.82$ & $21.88 \pm 42.00$ & $36.36 \pm 50.45$ & $7.69 \pm 27.74$ & $0.00 \pm 0.00$ & $0.00 \pm 0.00$ & 0.0014 \\
\hline Weight gain & $0.00 \pm 0.00$ & $56.25 \pm 50.40$ & $45.45 \pm 52.22$ & $61.54 \pm 50.64$ & $37.50 \pm 51.75$ & $66.67 \pm 57.74$ & 0.1531 \\
\hline
\end{tabular}

Table 6: Gender wise comparison of scales QLQ-C30 \& QLQ-H\&N35.

\begin{tabular}{|c|c|c|c|}
\hline Scales (QLQ-C30) & Male $(n=60)$ & Female $(n=13)$ & student $t$ Test $P$ value \\
\hline Global health status & $66.67 \pm 22.39$ & $69.23 \pm 30.12$ & 0.7266 \\
\hline Physical functioning & $89.22 \pm 10.49$ & $74.36 \pm 26.22$ & 0.0012 \\
\hline Role functioning & $90.00 \pm 16.58$ & $79.49 \pm 35.46$ & 0.1062 \\
\hline Emotional functioning & $75.28 \pm 24.35$ & $76.28 \pm 25.20$ & 0.8938 \\
\hline Cognitive functioning & $91.11 \pm 15.79$ & $76.92 \pm 32.30$ & 0.0206 \\
\hline Social functioning & $87.22 \pm 20.89$ & $83.33 \pm 34.69$ & 0.5949 \\
\hline Fatigue & $26.30 \pm 19.19$ & $37.61 \pm 27.04$ & 0.0787 \\
\hline Nausea and vomiting & $3.06 \pm 7.82$ & $5.13 \pm 12.52$ & 0.4435 \\
\hline Pain & $12.22 \pm 15.91$ & $42.31 \pm 32.36$ & $<0.0001$ \\
\hline Dyspnoea & $3.33 \pm 11.81$ & $2.56 \pm 9.25$ & 0.8263 \\
\hline Insomnia & $13.33 \pm 22.30$ & $30.77 \pm 28.74$ & 0.0179 \\
\hline Appetite loss & $21.67 \pm 29.96$ & $20.51 \pm 34.80$ & 0.9030 \\
\hline Constipation & $16.67 \pm 23.37$ & $7.69 \pm 14.62$ & 0.1893 \\
\hline Diarrhoea & $4.44 \pm 12.97$ & $2.56 \pm 9.25$ & 0.6222 \\
\hline Financial difficulties & $52.78 \pm 35.41$ & $58.97 \pm 38.86$ & 0.5756 \\
\hline \multicolumn{4}{|l|}{ QLQ H\&N-35 } \\
\hline Pain & $14.03 \pm 13.06$ & $35.26 \pm 36.03$ & 0.0005 \\
\hline Swallowing & $22.64 \pm 18.54$ & $33.33 \pm 29.27$ & 0.0964 \\
\hline Senses problems & $15.28 \pm 16.32$ & $35.90 \pm 23.42$ & 0.0003 \\
\hline Speech problems & $22.41 \pm 19.46$ & $46.15 \pm 29.34$ & 0.0006 \\
\hline Trouble with social eating & $27.64 \pm 25.83$ & $44.23 \pm 36.23$ & 0.0556 \\
\hline Trouble with social contact & $18.11 \pm 20.62$ & $29.74 \pm 36.07$ & 0.1166 \\
\hline Less sexuality & $16.94 \pm 27.01$ & $29.49 \pm 42.03$ & 0.1772 \\
\hline Teeth & $30.56 \pm 28.98$ & $41.03 \pm 36.40$ & 0.2634 \\
\hline Opening mouth & $37.78 \pm 35.50$ & $61.54 \pm 38.12$ & 0.0342 \\
\hline Dry mouth & $44.44 \pm 32.86$ & $61.54 \pm 38.12$ & 0.1028 \\
\hline Sticky saliva & $30.56 \pm 32.06$ & $30.77 \pm 37.17$ & 0.9832 \\
\hline Coughing & $15.56 \pm 18.88$ & $15.38 \pm 22.01$ & 0.9772 \\
\hline Felt ill & $24.44 \pm 25.94$ & $51.28 \pm 35.00$ & 0.0023 \\
\hline Pain killers & $20.00 \pm 40.34$ & $30.77 \pm 48.04$ & 0.4018 \\
\hline Nutritional supplements & $21.67 \pm 41.55$ & $38.46 \pm 50.64$ & 0.2081 \\
\hline Feeding tube & $1.67 \pm 12.91$ & $0.00 \pm 0.00$ & NA \\
\hline Weight loss & $21.67 \pm 41.55$ & $30.77 \pm 48.04$ & 0.4883 \\
\hline Weight gain & $50.00 \pm 50.42$ & $46.15 \pm 51.89$ & 0.8048 \\
\hline
\end{tabular}

all subset of the patients irrespective of their treatment plan received (Table 4).

In QOQ C-30 scale -comparing different interval of time elapsed since treatment, Global Health Status $(p=$ $0.03)$, Nausea \& Vomiting ( $p=0.0001)$, Pain $(p=0.0274)$ and Appetite loss ( $p=0.0355$ ) were found to be significant. However, after 3 months these symptoms scores became better. In QOQ H\&N-35 scale, swallowing ( $p$
$=0.001)$, speech $(p=0.004)$ and senses problems $(p$ $=0.0003)$, trouble with social eating $(p=0.0011)$ and contact $(p=0.012)$, less sexuality $(p=0.006)$, opening $(p=0.049)$ and dry mouth $(p=0.029)$, sticky saliva ( $p$ $=0.001)$, felt ill $(p=0.010)$ and weight loss $(p=0.001)$ were found to be significant, had a better score after 3 months (Table 5).

Depending upon the different gender categories - 
factors like Physical Functioning ( $p=0.0012)$, Cognitive functioning $(p=0.0206)$, Pain $(p=0.0001)$, Insomnia $(p=0.0179)$, Sense problems $(p=0.0003)$ and Speech problem $(p=0.0006)$ showed significant difference and male gender had better scores of all these factors as compared to female gender (Table 6).

As evident from Table 7, patients between age ranges of 21 to 40 had better score of global health status, physical functioning, pain, insomnia, dry mouth and pain killer. However, there was no statistically significant difference in any of the factors among all age subgroup.

\section{Discussion}

According to GLOBOCON-2018 head and neck cancer is the most common malignancy among males in
India. Lip and oral cavity cancers constitute $16 \%$ of all cancers in India. The habit of chewing betel nut leaves rolled with lime and tobacco (mixture known as "pan"), which results in prolonged carcinogen exposure to the oral mucosa, is thought to be the leading cause of oral cancer in Indian subcontinent [7]. The combined use of alcohol and tobacco may have a synergistic effect on carcinogenesis [8]. The high incidence of carcinoma of the buccal mucosa in our country is attributable to the extensive use of tobacco in various forms and the locally advanced cancers account for about $70 \%$ of the cases at the time of presentation [9].

In our study, Oral cavity (83.56\%) constitute the most common site followed by oropharynx (9.59\%) and $76.7 \%$ cases were in locally advanced stage (Stage III

Table 7: Age wise comparison of scales QLQ-C30 \& QLQ-H\&N35.

\begin{tabular}{|c|c|c|c|c|}
\hline Scales (QLQ-C30) & $21-40(n=14)$ & $41-60(n=46)$ & $>60(n=13)$ & ANOVA P value \\
\hline Global health status & $70.24 \pm 24.62$ & $67.21 \pm 24.68$ & $63.46 \pm 20.28$ & 0.7641 \\
\hline Physical functioning & $91.43 \pm 12.38$ & $85.07 \pm 16.76$ & $86.67 \pm 13.05$ & 0.4071 \\
\hline Role functioning & $88.10 \pm 23.05$ & $88.77 \pm 21.96$ & $85.90 \pm 17.80$ & 0.9138 \\
\hline Emotional functioning & $75.00 \pm 21.43$ & $76.81 \pm 22.77$ & $71.15 \pm 32.92$ & 0.7631 \\
\hline Cognitive functioning & $82.14 \pm 26.53$ & $89.49 \pm 19.36$ & $92.31 \pm 14.62$ & 0.3809 \\
\hline Social functioning & $85.71 \pm 28.39$ & $86.59 \pm 22.67$ & $87.18 \pm 23.72$ & 0.9871 \\
\hline Fatigue & $23.81 \pm 20.37$ & $29.71 \pm 21.60$ & $28.21 \pm 20.60$ & 0.6616 \\
\hline Nausea and vomiting & $7.14 \pm 12.60$ & $3.26 \pm 8.33$ & $0.00 \pm 0.00$ & 0.1034 \\
\hline Pain & $15.48 \pm 19.02$ & $17.03 \pm 23.44$ & $21.80 \pm 24.89$ & 0.7481 \\
\hline Dyspnoea & $2.38 \pm 8.91$ & $2.17 \pm 8.32$ & $7.69 \pm 19.97$ & 0.2916 \\
\hline Insomnia & $9.52 \pm 20.38$ & $17.39 \pm 25.08$ & $20.51 \pm 25.60$ & 0.4621 \\
\hline Appetite loss & $30.95 \pm 38.04$ & $18.84 \pm 29.52$ & $20.51 \pm 25.60$ & 0.4344 \\
\hline Constipation & $19.05 \pm 31.25$ & $13.77 \pm 20.58$ & $15.38 \pm 17.30$ & 0.7434 \\
\hline Diarrhoea & $2.38 \pm 8.91$ & $5.80 \pm 14.58$ & $0.00 \pm 0.00$ & 0.2801 \\
\hline Financial difficulties & $42.86 \pm 33.15$ & $57.25 \pm 36.96$ & $53.85 \pm 34.80$ & 0.4270 \\
\hline \multicolumn{5}{|l|}{ QLQ H\&N-35 } \\
\hline Pain & $14.88 \pm 12.31$ & $17.57 \pm 21.53$ & $21.80 \pm 24.66$ & 0.6837 \\
\hline Swallowing & $23.81 \pm 20.64$ & $23.91 \pm 20.83$ & $27.56 \pm 23.42$ & 0.8527 \\
\hline Senses problems & $22.62 \pm 23.21$ & $19.93 \pm 19.44$ & $11.54 \pm 12.52$ & 0.2846 \\
\hline Speech problems & $23.02 \pm 19.72$ & $28.02 \pm 23.86$ & $25.64 \pm 25.41$ & 0.7725 \\
\hline Trouble with social eating & $29.17 \pm 22.59$ & $30.98 \pm 30.21$ & $30.77 \pm 29.34$ & 0.9787 \\
\hline Trouble with social contact & $15.71 \pm 17.46$ & $19.86 \pm 24.32$ & $26.15 \pm 30.00$ & 0.5342 \\
\hline Less sexuality & $17.86 \pm 20.11$ & $22.46 \pm 34.64$ & $8.97 \pm 19.97$ & 0.3644 \\
\hline Teeth & $30.95 \pm 38.04$ & $32.61 \pm 27.66$ & $33.33 \pm 33.33$ & 0.9778 \\
\hline Opening mouth & $42.86 \pm 37.96$ & $42.03 \pm 37.47$ & $41.03 \pm 36.40$ & 0.9919 \\
\hline Dry mouth & $35.71 \pm 35.72$ & $48.55 \pm 34.22$ & $56.41 \pm 31.58$ & 0.2778 \\
\hline Sticky saliva & $28.57 \pm 28.81$ & $30.43 \pm 32.83$ & $33.33 \pm 38.49$ & 0.9317 \\
\hline Coughing & $14.29 \pm 17.12$ & $15.94 \pm 20.77$ & $15.38 \pm 17.30$ & 0.9619 \\
\hline Felt ill & $26.19 \pm 23.31$ & $28.99 \pm 31.90$ & $33.33 \pm 27.22$ & 0.8201 \\
\hline Pain killers & $14.29 \pm 36.31$ & $19.57 \pm 40.11$ & $38.46 \pm 50.64$ & 0.2668 \\
\hline Nutritional supplements & $14.29 \pm 36.31$ & $26.09 \pm 44.40$ & $30.77 \pm 48.04$ & 0.5813 \\
\hline Feeding tube & $0.00 \pm 0.00$ & $0.00 \pm 0.00$ & $7.69 \pm 27.74$ & 0.0984 \\
\hline Weight loss & $21.43 \pm 42.58$ & $21.74 \pm 41.70$ & $30.77 \pm 48.04$ & 0.7877 \\
\hline Weight gain & $42.86 \pm 51.36$ & $54.35 \pm 50.36$ & $38.46 \pm 50.64$ & 0.5300 \\
\hline
\end{tabular}


\& IV). Males constitute $82.19 \%$ and females constitute $17.8 \%$ of the study sample. $63 \%$ patients were in age group 41-60 years. 72 patients were married whereas one patient was unmarried. The treatment plan of surgery followed by radiation was received by $67.49 \%$ patients.

When the comparison was made on the basis of stage (early stage versus advanced stage), overall, there was no significant difference in different parameters except physical functioning, role functioning, emotional functioning, dry mouth and felt ill scores which were better in early stage tumors. Elumelu, et al. [10] evaluated EORTC QLQ-C30 results according to disease stage in their patients with head and neck cancer, and found that mean scores for global health status and all functional scales were significantly lower for late-stage (III and IV) patients than for early-stage (I and II) patients. They also observed that late-stage patients had significantly higher scores for all symptom scales/items than early-stage patients.

In our study, irrespective of the type of treatment received either RT alone ( $n=3)$, Concurrent Chemo-RT ( $n$ $=8)$, Surgery followed by Chemo-RT ( $n=12$ ) or, Surgery followed by RT ( $n=50)$; taking both scales in account, nutritional supplement is the only parameter found on QLQ-H\&N35 which showed significance ( $p=0.0572$ ). Nausea and vomiting score were a bit more in surgery followed by Radiotherapy along with concurrent chemotherapy. Hammerlid E, et al. also have shown in their study that the combined modality of the treatment had a negative impact on the individuals QoL [11].

De Graeff, et al. [12] prospectively evaluated changes in QoL for 107 patients with squamous cell carcinomas of the head and neck who underwent post-operative RT. The authors used the EORTC QLQ-C30 as well as the QLQ-H\&N35, a questionnaire specific for patients with head and neck cancer and applied these instruments before RT and 6, 12, 24, and 36 months after RT. Their results for EORTC QLQ-C30 at 6 months post-RT revealed significant deterioration in the scores for physical functioning, role functioning, emotional functioning, and fatigue; however, by 6 months later (12 months after RT), all these scores had improved significantly.

In our study also, with time passes after treatment, quality of life parameter improved in both scale EORTC QLQ C-30 and EORTC H\&N-35. In QLQ C-30 Scale, Global Health Status, Nausea \& Vomiting, Pain and Appetite loss were found to be significant, should be taken care off during the treatment, so it will have improved score at the time of first follow up within six weeks of the completion of their treatment itself. However, after 3 months, scores of these symptoms became better spontaneously. In QOQ H\&N-35 scale also, swallowing, speech and senses problems, trouble with social eating and contact, less sexuality, opening and dry mouth, sticky saliva, felt ill and weight loss were significant, had a better score after 3 months only. It shows that all above significant factors must be kept in the mind prior to start the treatment to improve quality of life during and after the completion of treatment of head and neck cancer patients.

Elumelu, et al. [10] investigated 100 patients with head and neck cancer and applied the EORTC QLQ-C30 and QLQ-H\&N35 (mentioned above) at the beginning and end of RT. At the end of RT, they observed that in females, mean scores for role functioning, cognitive functioning, social functioning, dyspnea and constipation were higher than those for males, whereas males had higher mean scores for fatigue, pain, insomnia, appetite loss, diarrhoea and financial difficulties. Males and females had almost equal scores for global health status, physical functioning, emotional functioning, and nausea/vomiting. In our study, global health status, physical functioning, Cognitive functioning, pain, insomnia, swallowing, sense and speech problem, opening mouth, dry mouth and felt ill scores were better in male patients than females.

In Elumelu, et al. [10] study, low socioeconomic status has a negative influence on their HR-QoL. In our study, overall quality of life had not changed significantly on the basis of different socioeconomic strata. Yes, financial difficulties score was higher in low socioeconomic group. Physical functioning, Role functioning, Emotional functioning, Cognitive functioning scores a little bit better in Middle/High socioeconomic group.

Overall, in our study, taking all group of the patients into account either of early or advanced stage and received either single and combined modalities of the treatment, apart from above mentioned significant factors, nutritional supplements has been emerged as a key factor. Therefore, it always can be suggested that nutritional supplementation must be kept in mind prior to start and during the treatment of any head and neck cancer patients which would lead to definite improvement in quality of life.

One important point we must emphasize that since this study is a cross sectional study which is carried out in a short time period. It is always suggested to have randomized controlled trial and multicentric studies with large number of the patients to elaborate such results.

\section{Conclusion}

$\mathrm{H} \& \mathrm{~N}$ cancer has a significant impact on individuals QoL. Assessment of quality of life should receive adequate attention in all such patients. Nutritional supplements are the parameter found to be significant in all head and neck cancer patients and should be taken care prior, during and after the treatment. Other factors like Physical functioning, pain, Cognitive functioning, Insomnia, speech problems, senses problems, opening and dry mouth, trouble in social contact and eating, felt 
ill are also found to be significant during course of the treatment which needs clinician's attention, its proper care and counselling to improve QoL in such patients.

\section{Ethical Clearance and Conflicts of Interest}

This is a cross-sectional study conducted at Paras HMRI Hospital, Patna (India) among radically treated head and neck cancer patients attending radiation oncology OPD for follow up and it is based on questionnaire obtained from EORTC. There was no change in the treatment protocol received by the head and neck cancer patients according to their stage of cancer. Since, this is a questionnaire-based study; ethical committee clearance was not required. This study has been approved and allowed by the Head of the Institute. We do not have any conflicts of interest.

\section{Highlights}

- H\&N cancer has a significant impact on individuals QoL.

- Assessment of Quality of Life in Radically Treated Head \& Neck Cancer Patients.

- Nutritional supplement is the significant factor in all subsets of treated head and neck cancer patients.

- Special precaution of this sole factor "Nutritional Supplement" may significantly improve quality of life in these patients.

\section{Source(s) of support}

None.

\section{References}

1. Sanghvi LD, Rao DN, Joshi S (1989) Epidemiology of head and neck cancers. Semin Surg Oncol 5: 305-309.
2. Lo Nigro C, Denaro N, Merlotti A, Merlano M (2017) Head and neck cancer: Improving outcomes with a multidisciplinary approach. Cancer Manag Res 9: 363-371.

3. Jaydip Biswas (2014) Debunk the myths: Oncologic misconceptions. Indian J Med Res 139: 185-187.

4. Rogers SN, Semple C, Babb M, Humphris G (2016) Quality of life consideration in head and neck cancer: United Kingdom National multidisciplinary guidelines. J Laryngol Otol 130: S49-S52.

5. Singer S, Wollbruck D, Wulke C, Dietz A, Klemm E, et al. (2009) Validation of the EORTC QLQ-C30 and EORTC QLQ-H\&N35 in patients with laryngeal cancer after surgery. Head Neck 31: 64-76.

6. Chaukar DA, Das AK, Deshpande MS, Pai PS, Pathak KA, et al. (2005) Quality of life of head and neck cancer patient: Validation of the European organization for research and treatment of cancer QLQ-C30 and European organization for research and treatment of cancer QLQ-H\&N35 in Indian patients. Indian J Cancer 42: 178-184.

7. Sharma DC (2003) Betel quid and areca nut are carcinogenic without tobacco. Lancet Oncol 4: 587.

8. Lambert R, Sauvaget C, de Camargo Cancela M, Sankaranarayanan $R$ (2011) Epidemiology of cancer from the oral cavity and oropharynx. Eur J Gastroenterol Hepatol 23: 633-641.

9. Ramesh Shankar (2012) Consensus document for management of buccal mucosa cancer.

10. Elumelu TN, Adenipekun AA, Abdus-salam AA, Bojude AD, Campbell OB (2011) Quality of life in patients with head and neck cancer on radiotherapy treatment at Ibadan. Researchers 3: 1-10.

11. Hammerlid E, Silander E, Hornestam L, Sullivan M (2001) Health-related quality of life three years after diagnosis of head and neck cancer--a longitudinal study. Head Neck 23: 113-125.

12. De Graeff A, de Leeuw JR, Ros WJ, Hordiik GJ, Bliiham $\mathrm{GH}$, et al. (2000) Long-term quality of life of patients with head and neck cancer. Laryngoscope 110: 98-106. 